\title{
BIFURCATION PHENOMENA ASSOCIATED TO THE $p$-LAPLACE OPERATOR
}

\author{
MOHAMMED GUEDDA AND LAURENT VERON
}

\begin{abstract}
We determine the structure of the set of the solutions $u$ of $-\left(\left|u_{x}\right|^{p-2} u_{x}\right)_{x}+f(u)=\lambda|u|^{p-2} u$ on $(0,1)$ such that $u(0)=u(1)=0$, where $p>1$ and $\lambda \in \mathbf{R}$. We prove that the solutions with $k$ zeros are unique when $1<p \leq 2$ but may not be so when $p>2$.
\end{abstract}

0. Introduction. In this article we study the structure of the set $E_{\lambda}$ of the solutions of the following nonlinear eigenvalue problem

$$
\left\{\begin{array}{l}
-\left(\left|u_{x}\right|^{p-2} u_{x}\right)_{x}+f(u)=\lambda|u|^{p-2} u \text { in }(0,1), \\
u(0)=u(1)=0
\end{array}\right.
$$

where $p>1, \lambda$ is a real number and $f$ is a $C^{1}$ real-valued odd function such that

$$
r \mapsto g(r)=f(r) /\left(|r|^{p-2} r\right)
$$

is increasing on $(0,+\infty)$ with limits 0 at 0 and $+\infty$ at infinity. We first investigate the unperturbed eigenvalue problem

$$
\left\{\begin{array}{l}
-\left(\left|v_{x}\right|^{p-2} v_{x}\right)_{x}=\lambda|v|^{p-2} v \quad \text { in }(0,1) \\
v(0)=v(1)=0
\end{array}\right.
$$

By means of an elementary integration process we prove that $(0.3)$ admits a nontrivial solution if and only if

$$
\lambda=\lambda_{k}=k^{p}(p-1)\left[2 \int_{0}^{1} \frac{d t}{\left(1-t^{p}\right)^{1 / p}}\right]^{p}, \quad k \in \mathbf{N}^{*} .
$$

Moreover to each $\lambda_{k}$ is associated a one-dimensional eigenspace generated by a function $\omega_{k}$ with exactly $k-1$ zeros in $(0,1)$. Concerning the equation $(0.1)$ we prove that each $\lambda_{k}$ is a point of bifurcation as in the semilinear case $(p=2)$. More precisely we define for $k \in \mathbf{N}^{*}$

$$
S_{k}=\{\varphi \in C: \varphi \text { has exactly } k-1 \text { simple zeros in }(0,1)\},
$$

where $C=\left\{\varphi \in C^{1}([0,1]): \varphi(0)=\varphi(1)=0\right\}$ and

$$
S_{k}^{+}=\left\{\varphi \in S_{k}: \varphi_{x}(0)>0\right\}, \quad S_{k}^{-}=-S_{k}^{+} .
$$

As $\lambda_{1}$ is defined as the best Poincaré constant in $W_{0}^{1, p}(0,1)$, that is,

$$
\operatorname{Inf}\left\{\int_{0}^{1}\left|v_{x}\right|^{p} d x: v \in W_{0}^{1, p}(0,1), \int_{0}^{1}|v|^{p} d x=1\right\},
$$

Received by the editors July 4, 1987 and, in revised form, October 12, 1987.

1980 Mathematics Subject Classification (1985 Revision). Primary 35J60; Secondary 34C30. 
it is clear that $E_{\lambda}$ is reduced to the zero function when $\lambda \leq \lambda_{1}$.

When $1<p \leq 2$ we prove that the configuration of $E_{\lambda}$ is exactly the same as in the case $p=2[\mathbf{1}]$, that is,

$$
E_{\lambda}=\left\{0, \pm u_{l}, l=1, \ldots, k: u_{l} \in S_{l}^{+}\right\} .
$$

When $p>2$ the structure of $E_{\lambda}$ can be quite a bit more complicated for large values of $\lambda$. Let $h$ be the inverse function of $g$ and $F(r)=\int_{0}^{r} f(s) d s$; we define

$$
\alpha(\lambda)=\left(\frac{\lambda}{p-1} h^{p}(\lambda)-\frac{p}{p-1} F(h(\lambda))\right)^{1 / p}
$$

and

$$
x(\lambda)=\int_{0}^{h(\lambda)} \frac{d s}{\left(\alpha^{p}(\lambda)+p F(s) /(p-1)-\lambda s^{p} /(p-1)\right)^{1 / p}} ;
$$

and $\lambda \mapsto x(\lambda)$ is a decreasing positive function defined on $(0,+\infty)$. If $\lambda_{k}<\lambda \leq$ $\lambda_{k+1}$ we then have

$$
E_{\lambda}=\{0\} \cup\left\{ \pm u_{1}\right\} \bigcup_{p=2}^{k}\left\{ \pm E_{\lambda}^{l}\right\}
$$

where $u_{1} \in S_{1}^{+}$and $E_{\lambda}^{l} \subset S_{l}^{+}$such that

(i) $E_{\lambda}^{l}$ contains only one element if $2 l x(\lambda) \geq 1$,

(ii) $E_{\lambda}^{l}$ is diffeomorphic to $[0,1]^{l-1}$ if $0<2 l x(\lambda)<1$. In case (ii) the elements of $E_{\lambda}^{l}$ are constant with value $(-1)^{j+1} h(\lambda)$ on $l$ closed and disconnected subintervals $I_{j} \subset(0,1), j=1, \ldots, l$, with total length $1-2 l x(\lambda)$.

1. The eigenvalue problem. For $p>1$ we consider the following eigenvalue problem

$$
\left\{\begin{array}{l}
-\left(\left|v_{x}\right|^{p-2} v_{x}\right)_{x}=\lambda|v|^{p-2} v \quad \text { in }(0,1) \\
v(0)=v(1)=0
\end{array}\right.
$$

and let $S$ be the subset of $W_{0}^{1, p}(0,1) \times \mathbf{R}$ of all the $(v, \lambda), v \neq 0$, satisfying (1.1).

THEOREM 1.1. There exists a unique sequence of functions $v_{k} \in S_{k}^{+}, k \in \mathbf{N}^{*}$, with maximal value 1 on $(0,1)$ such that

$$
S=\left\{\left(\mu v_{k}, \lambda_{k}\right): k \in \mathbf{N}^{*}\right\},
$$

where $\mu$ is any nonzero real number and

$$
\lambda_{k}=k^{p} \lambda_{1}=k^{p}(p-1)\left[2 \int_{0}^{1} \frac{d t}{\left(1-t^{p}\right)^{1 / p}}\right]^{p} .
$$

Moreover the following holds for $m=0, \ldots, k-1$ :

$$
v_{k}(x)=(-1)^{m} v_{1}(k x-m), \quad m / k \leq x \leq(m+1) / k .
$$

Before giving the proof it must be noticed that this result is partially contained in [5], in particular formula (1.4).

PROOF. It is clear from $(1.1)$ and $v \in C^{0}([0,1])$ and then $v \in C^{1}([0,1])$ when $p>2$ or $v \in C^{2}([0,1])$ when $1<p \leq 2$ (the complete regularity, due to Otani [5], will be given in Remark 1.1). 
Step 1. If $(v, \lambda) \in S$ then $v_{x}(0) \neq 0$ and $\lambda>0$. Multiplying (1.1) by $v$ and integrating over $(0,1)$ yields

$$
\int_{0}^{1}\left|v_{x}\right|^{p} d x=\lambda \int_{0}^{1} v^{p} d x
$$

Hence necessarily $\lambda>0$. Multiplying (1.1) by $v_{x}$ and integrating over $(0, x), 0<$ $x<1$, yields the energy estimate

$$
(p-1)\left|v_{x}(x)\right|^{p}+\lambda|v(x)|^{p}=(p-1)\left|v_{x}(0)\right|^{p}+\lambda|v(0)|^{p} .
$$

As $v(0)=0$ we need $v_{x}(0) \neq 0$ in order to have a nonzero $v$.

Step 2. The explicit construction. Assume $v$ is a nonzero solution with $v_{x}(0)=$ $\alpha>0$ for example. Then $v_{x}>0$ on $\left[0, x_{0}\right)$ for some $x_{0} \in(0,1)$ and

$$
v_{x}(x)=\left(\alpha^{p}-\frac{\lambda}{p-1}(v(x))^{p}\right)^{1 / p}
$$

on $\left[0, x_{0}\right]$, from (1.6), which gives

$$
x=\int_{0}^{v(x)} \frac{d t}{\left(\alpha^{p}-\lambda t^{p} /(p-1)\right)^{1 / p}} .
$$

Moreover this formula remains valid as long as $v(x)$ remains smaller than the first positive zero of the function

$$
r \mapsto \varphi(\alpha, r)=\alpha^{p}-\lambda r^{p} /(p-1)
$$

which is $S(\alpha)=((p-1) / \lambda)^{1 / p} \alpha$. As $S(\alpha)$ is simple we define $\theta(\alpha)$ by

$$
\theta(\alpha)=\int_{0}^{S(\alpha)} \frac{d t}{\left(\alpha^{p}-\lambda t^{p} /(p-1)\right)^{1 / p}} .
$$

Moreover $v(\theta(\alpha))=S(\alpha)$ and $v_{x}(\theta(\alpha))=0$. As $\alpha^{p}=\lambda S^{p}(\alpha) /(p-1)$ we get

$$
\theta(\alpha)=\theta_{\lambda}=C\left(\frac{p-1}{\lambda}\right)^{1 / p}, \quad C=\int_{0}^{1} \frac{d s}{\left(1-s^{p}\right)^{1 / p}}
$$

From (1.6) the function $v$ is decreasing on some interval $\left[\theta_{\lambda}, \Theta\right)$, so we get

$$
x-\theta_{\lambda}=-\int_{v(x)}^{S(\alpha)} \frac{d t}{\left[(\lambda /(p-1))\left(S^{p}(\alpha)-t^{p}\right)\right]^{1 / p}},
$$

or

$$
x-\theta_{\lambda}=-\int_{v(x)}^{S(\alpha)} \frac{d t}{\left(\alpha^{p}-\lambda t^{p} /(p-1)\right)^{1 / p}} ;
$$

and this formula remains valid as long as $v$ is decreasing, in particular as long as $v$ is positive. If $x_{1} \in\left(0, \theta_{\lambda}\right)$ and $x_{2}=2 \theta_{\lambda}-x_{1}$ then

$$
x_{1}=\int_{0}^{v\left(x_{1}\right)} \frac{d t}{(\varphi(\alpha, t))^{1 / p}}, \quad \theta_{\lambda}-x_{1}=-\int_{v\left(x_{2}\right)}^{S(\alpha)} \frac{d t}{(\varphi(\alpha, t))^{1 / p}}
$$

and $v\left(x_{1}\right)=v\left(x_{2}\right)$. As a consequence $x=\theta_{\lambda}$ is an axis of symmetry for the restriction of $v$ to $\left[0,2 \theta_{\lambda}\right]$ and $x=2 \theta_{\lambda}$ is a center of symmetry for the restriction of 
$v$ to $\left[0,4 \theta_{\lambda}\right]$. Hence the function $v$ is $4 \theta_{\lambda}$-periodic on $[0,+\infty)$. The necessary and sufficient condition for the restriction of $v$ to $[0,1]$ to be a solution of $(1.1)$ is then

$$
1 / 2 \theta_{\lambda} \in \mathbf{N}^{*}
$$

which means (1.3). As for the number of zeros of $v$ in $(0,1)$ it is given by $1 / 2 \theta_{\lambda}-1$. Using the homogeneity of (1.1) we get the desired result as the uniqueness is a consequence of the construction of $v$.

REMARK 1.1. Existence and uniqueness of the first positive normalized eigenfunction of $-\operatorname{div}\left(|D .|^{p-2} D\right.$. $)$ in $W_{0}^{1, p}(\Omega)$ have been obtained by De Thelin in the radial case when $\Omega$ is a ball $[\mathbf{7}]$ and Guedda-Veron for general $\Omega$ with a connected $C^{2}$ boundary [4].

As for the regularity of $v$ we have

$$
v \in C^{\alpha}([0,1]) \cap C^{\langle p\rangle}([0,1] \backslash Z)
$$

where $Z=\left\{x \in(0,1): v_{x}(x)=0\right\}, \alpha=\min (\langle(2-p) /(p-1)\rangle+1,\langle p\rangle)$ and $\langle r\rangle=+\infty$ if $r \in 2 \mathbf{N}^{*}$ or $\langle r\rangle=\min \left\{n: n \in \mathbf{N}^{*}, n \geq r\right\}$ if not.

REMARK 1.2. We have the following Poincaré type relation

$$
\lambda_{1}=\operatorname{Inf}\left\{\int_{0}^{1}\left|u_{x}\right|^{p} d x / \int_{0}^{1}|u|^{p} d x: u \in W_{0}^{1, p}(0,1) \backslash\{0\}\right\}
$$

and the infimum is achieved for $u=v_{1}$.

2. The bifurcation phenomena. In this section we consider the following equation

$$
\left\{\begin{array}{l}
-\left(\left|u_{x}\right|^{p-2} u_{x}\right)_{x}+f(u)=\lambda|u|^{p-2} u \text { in }(0,1), \\
u(0)=u(1)=0
\end{array}\right.
$$

where $p>1$ and $\lambda \in \mathbf{R}$. As for $f$ we first assume that

$$
f \text { is a } C^{1} \text { odd function, }
$$$$
s \mapsto f(s) / s^{p-1} \text { is strictly increasing on }(0,+\infty) \text { with limit } 0 \text { at } 0 \text {, }
$$

We then define

$$
\lim _{s \rightarrow+\infty} f(s) / s^{p-1}=+\infty
$$

where $F(s)=\int_{0}^{s} f(t) d t$. For $\lambda>0$ we shall also consider the following hypothesis:

$$
(p-1)\left(H^{\prime}(s)\right)^{2}-p H(s) H^{\prime \prime}(s) \geq 0 \text { for any } s \in[0, h(\lambda)] .
$$

Let $E_{\lambda}$ be the set of all the solutions of $(2.1)$ in $W_{0}^{1, p}(0,1)$ and $\lambda_{k}$ be defined by (1.3). When $1<p \leq 2$ the structure of $E_{\lambda}$ is exactly the same as in the case $p=2$. 
THEOREM 2.1. Assume $1<p \leq 2$ and (2.2)-(2.7). Then

(i) if $\lambda \leq \lambda_{1}, E_{\lambda}=\{0\}$, and

(ii) if $\lambda_{k}<\lambda \leq \lambda_{k+1}$ for some $k \in \mathbf{N}^{*}$

$$
E_{\lambda}=\left\{0, \pm u_{1}, \ldots, \pm u_{k}\right\},
$$

where $u_{l} \in S_{l}^{+}$for $l=1, \ldots, k$.

REMARK 2.1. The assumption (2.7), which is equivalent to the fact that $s \mapsto$ $H^{p-1}(s) / H^{\prime p}(s)$ is nondecreasing on $[0, h(\lambda)]$, is essential for uniqueness but not for existence. In the particular case where $f(r)=|r|^{q-1} r$ with $q>p-1$ then $h(\lambda)=\lambda^{1 /(q+1-p)}, H(s)=\lambda s^{p}-p s^{q+1} /(q+1)$ and $(2.7)$ is satisfied.

PROOF OF THEOREM 2.1. As in Theorem 1.1 it is clear that any solution of $(2.1)$ in $W_{0}^{1, p}(0,1)$ is continuous and at least $C^{2}$ (remember that $1<p \leq 2$ ). Multiplying the equation by $u$ yields

$$
\int_{0}^{1}\left|u_{x}\right|^{p} d x+\int_{0}^{1} u f(u) d x=\lambda \int_{0}^{1}|u|^{p} d x .
$$

From Remark 1.2 a nonzero solution of (2.1) can exist only if $\lambda>\lambda_{1}$, which will be assumed in the sequel.

Step 1. If $u$ is a nonzero solution of $(2.1)$ then $u_{x}(0) \neq 0$. Although it is a consequence of a general result due to Franchi, Lanconelli and Serrin, we give here a direct proof which also works when $p>2$. Multiplying (2.1) by $u_{x}$ yields the energy relation

$$
\begin{aligned}
& -\frac{p-1}{p}\left|u_{x}(x)\right|^{p}+F(u(x))-\frac{\lambda}{p}|u(x)|^{p} \\
& \quad=-\frac{p-1}{p}\left|u_{x}(0)\right|+F(u(0))-\frac{\lambda}{p}|u(0)|^{p} .
\end{aligned}
$$

If we assume that $u_{x}(0)=0$ we get

$$
\left|u_{x}(x)\right|^{p}=\frac{1}{p-1}\left(p F(u(x))-\lambda|u(x)|^{p}\right) .
$$

As the function $x \rightarrow p F(x)-\lambda|x|^{p}$ is negative on $(-\rho, \rho) \backslash\{0\}, u_{x}$ is always 0 and $u \equiv 0$.

Step 2. The explicit construction. Without any loss of generality we assume $u_{x}(0)=\alpha>0$. Hence $u$ is increasing on some interval $\left[0, x_{0}\right]$ and from (2.10) we get

$$
u_{x}^{p}(x)=\alpha^{p}+\frac{p}{p-1} F(u(x))-\frac{\lambda}{p-1} u^{p}(x)
$$

which gives $u$ as the inverse function of a $p$-elliptic integral

$$
x=\int_{0}^{u(x)} \frac{d t}{\left(\alpha^{p}+p F(t) /(p-1)-\lambda t^{p} /(p-1)\right)^{1 / p}}
$$

on $\left[0, x_{0}\right]$. Moreover this formula remains valid as long as $u(x)$ is smaller than the first positive zero of

$$
r \mapsto \Psi(\alpha, r)=\alpha^{p}+\frac{p}{p-1} F(r)-\frac{\lambda}{p-1}|r|^{p} .
$$


But the function $\Psi(\alpha, \cdot)$ is decreasing in $[0, h(\lambda)]$ and increasing on $[h(\lambda),+\infty)$; hence there are three possibilities.

Case 1. $\alpha^{p}>\lambda h^{p}(\lambda) /(p-1)-p F(h(\lambda)) /(p-1)=\alpha^{p}(\lambda)$.

In that case the function $r \mapsto \int_{0}^{r} d s /(\Psi(\alpha, s))^{1 / p}$ is an increasing $C^{2}$ diffeomorphism from $\mathbf{R}^{+}$onto $\mathbf{R}^{+}$and it is the same with $u$ defined by (2.13) which cannot belong to $E_{\lambda}$.

Case 2. $\alpha^{p}=\alpha^{p}(\lambda)$.

In that case $h(\lambda)$ is a double zero for $\Psi(\alpha, \cdot)$, and as $1<p \leq 2$

$$
\int_{0}^{h(\lambda)} d s /(\Psi(\alpha, s))^{1 / p}=+\infty
$$

As in Case 1 the function $r \mapsto \int_{0}^{r} d s /(\Psi(\alpha, s))^{1 / p}$ is a $C^{2}$ diffeomorphism from $[0, h(\lambda))$ onto $\mathbf{R}^{+}$and $u$ cannot belong to $E_{\lambda}$.

Case 3. $\alpha^{p}<\alpha^{p}(\lambda)$.

In that case $\Psi(\alpha, \cdot)$ admits a simple zero $S(\alpha)$ in $(0, h(\lambda))$. As $(\partial \Psi / \partial r)(\alpha, S(\alpha))$ $\neq 0, r \mapsto(\Psi(\alpha, r))^{-1 / p}$ is integrable on $(0, S(\alpha))$ and we define

$$
\theta(\alpha)=\int_{0}^{S(\alpha)} \frac{d s}{(\Psi(\alpha, s))^{1 / p}} .
$$

Relation (2.13) remains valid on $[0, \theta(\alpha)]$ and we have

$$
u(\theta(\alpha))=S(\alpha), \quad u_{x}(\theta(\alpha))=0 .
$$

Using the energy relation at $\theta(\alpha)$ we have

or

$$
\frac{p-1}{p}\left|u_{x}(x)\right|^{p}=\frac{\lambda}{p} S^{p}(\alpha)-F(S(\alpha))-\left(\frac{\lambda}{p} u^{p}(x)-F(u(x))\right)
$$

$$
\left|u_{x}(x)\right|^{p}=\alpha^{p}+\frac{p}{p-1} F(u(x))-\frac{\lambda}{p-1} u^{p}(x) .
$$

Hence $u$ is decreasing on some interval $[\theta(\alpha), \Theta]$ and we have

$$
x-\theta(\alpha)=-\int_{u(x)}^{S(\alpha)} \frac{d s}{(\Psi(\alpha, s))^{1 / p}} .
$$

This formula remains valid as long as $u$ is decreasing, and as in $\S 1 x=\theta(\alpha)$ is an axis of symmetry for the restriction of $u$ to $[0,2 \theta(\alpha)]$ and $x=2 \theta(\alpha)$ is a center of symmetry for the restriction of $u$ to $[0,4 \theta(\alpha)]$; the necessary and sufficient condition for $u$ to be a solution of $(2.1)$ is that

$$
1 / 2 \theta(\alpha) \in \mathrm{N}^{*} \text {. }
$$

Step 3. The function $\alpha \mapsto S(\alpha)$ is convex, increasing on $[0, \alpha(\lambda))$. We have $\Psi(\alpha, S(\alpha))=0$ and $(\partial \Psi / \partial r)(\alpha, S(\alpha)) \neq 0$. By the implicit function theorem $\alpha \mapsto S(\alpha)$ is $C^{2}$. We also have

$$
\frac{d}{d \alpha}(\Psi(\alpha, S(\alpha)))=\frac{\partial \Psi}{\partial \alpha}(\alpha, S(\alpha))+\frac{\partial \Psi}{\partial r}(\alpha, S(\alpha)) \frac{d S}{d \alpha}(\alpha)
$$

which gives

$$
\frac{d S}{d \alpha}(\alpha)=\frac{(p-1) \alpha^{p-1}}{\lambda S^{p-1}(\alpha)-f(S(\alpha))}=\frac{p(p-1) \alpha^{p-1}}{H^{\prime}(S(\alpha))} .
$$


As $S(\alpha)<h(\lambda), \alpha \mapsto S(\alpha)$ is increasing on $[0, \alpha(\lambda))$. Moreover

$$
\frac{d^{2} S}{d \alpha^{2}}(\alpha)=p(p-1) \frac{(p-1) \alpha^{p-2} H^{\prime}(S(\alpha))-\alpha^{p-1} H^{\prime \prime}(S(\alpha)) d S / d \alpha}{\left(H^{\prime}(S(\alpha))\right)^{2}}
$$

Using (2.20) and the definition of $S(\alpha)$ and $H$ we get

$$
\frac{d^{2} S}{d \alpha^{2}}(\alpha)=p(p-1) \alpha^{p-2} \frac{(p-1)\left(H^{\prime}(S(\alpha))\right)^{2}-p H(S(\alpha)) H^{\prime \prime}(S(\alpha))}{\left(H^{\prime}(S(\alpha))\right)^{3}}
$$

From (2.7) we deduce $d^{2} S(\alpha) / d \alpha^{2} \geq 0$.

Step 4. The function $\alpha \mapsto \theta(\alpha)$ is continuous increasing on $[0, \alpha(\lambda))$. For $t \in[0, \alpha]$ the function $s \mapsto \Psi(t, s)$ admits a first positive zero at $S(t)$ which means

$$
t^{p}+\frac{p}{p-1} F(S(t))-\frac{\lambda}{p-1} S^{p}(t)=0 \quad \text { and } \quad \Psi(\alpha, S(t))=\alpha^{p}-t^{p} .
$$

Taking $t$ as a new variable in (2.15) we get

$$
\theta(\alpha)=\int_{0}^{\alpha} \frac{d S}{d t}(t) \frac{d t}{\left(\alpha^{p}-t^{p}\right)^{1 / p}}
$$

or

$$
\theta(\alpha)=\int_{0}^{1} \frac{d S}{d t}(\alpha \sigma) \frac{d \sigma}{\left(1-\sigma^{p}\right)^{1 / p}} .
$$

As $d s / d t$ is increasing and $C^{1}$ on $[0, \alpha(\lambda))$, it is the same with $\alpha \mapsto \theta(\alpha)$.

Step 5. End of the proof. As $\lim _{\alpha \downarrow 0} S(\alpha)=0$ and $\lim _{\alpha \downarrow 0} F(S(\alpha)) / S^{p}(\alpha)=0$ we get

$$
S(\alpha) \underset{\alpha \downarrow 0}{\sim} \alpha\left(\frac{p-1}{\lambda}\right)^{1 / p}
$$

which implies

$$
\lim _{\alpha \downarrow 0} \frac{d S}{d \alpha}(\alpha)=\left(\frac{p-1}{\lambda}\right)^{1 / p}
$$

and

$$
\lim _{\alpha \downarrow 0} \theta(\alpha)=\left(\frac{p-1}{\lambda}\right)^{1 / p} \int_{0}^{1} \frac{d \sigma}{\left(1-\sigma^{p}\right)^{1 / p}}=\frac{1}{2}\left(\frac{\lambda_{1}}{\lambda}\right)^{1 / p}
$$

For the other bound we have $\lim _{\alpha \downarrow \alpha(\lambda)} S(\alpha)=h(\lambda)$. As $h(\lambda)$ is just a double zero for $\Psi(\alpha(\lambda), r)$, there exists a continuous and bounded function $\varphi$ on $[0, \alpha(\lambda)]$ such that

$$
\Psi(\alpha(\lambda), r)=(h(\lambda)-r)^{2} \varphi(r) .
$$

Moreover

$$
\begin{aligned}
\int_{0}^{S(\alpha)}(\Psi(\alpha, t))^{-1 / p} d t & >\int_{0}^{S(\alpha)}(\Psi(\alpha(\lambda), t))^{-1 / p} d t \\
& =\int_{0}^{S(\alpha)}(h(\lambda)-t)^{-2 / p}(\varphi(t))^{-1 / p} d t
\end{aligned}
$$

As $1<p \leq 2$ we get

$$
\lim _{\alpha \uparrow \alpha(\lambda)} \theta(\alpha)=\int_{0}^{h(\lambda)}(h(\lambda)-t)^{-2 / p}(\varphi(t))^{-1 / p} d t=+\infty
$$


As a consequence $\alpha \mapsto \theta(\alpha)$ is an increasing diffeomorphism from $(0, \alpha(\lambda))$ onto $\left(\frac{1}{2}\left(\lambda_{1} / \lambda\right)^{1 / p},+\infty\right)$ and $1 / 2 \theta(\alpha)$ a decreasing diffeomorphism from $(0, \alpha(\lambda))$ onto $\left(0,\left(\lambda / \lambda_{1}\right)^{1 / p}\right)$. If we assume that $\lambda_{k}<\lambda \leq \lambda_{k+1}$ for some $k \in \mathbf{N}^{*}$ there exist exactly $k$ integers $l=1, \ldots, k$ and $k$ positive real numbers $\alpha_{l}$ such that $1 / 2 \theta\left(\alpha_{l}\right)=l$. If $u_{l}$ is the solution of the initial value problem

$$
\left\{\begin{array}{l}
-\left(\left|u_{l x}\right|^{p-2} u_{l x}\right)_{x}+f\left(u_{l}\right)=\lambda\left|u_{l}\right|^{p-2} u_{l} \quad \text { on }(0,1) \\
u_{l}(0)=0, \quad u_{l x}(0)=\alpha_{l}
\end{array}\right.
$$

then $u_{l}(1)=0, u_{l} \in S_{l}^{+}$. We get the result in considering $-u_{l}, l=1, \ldots, k$.

REMARK 2.2. If we represent the bifurcation diagram $\left(\lambda, u_{\lambda}\right)$ then there exists no secondary bifurcation along the branches of solutions in $S_{k}^{ \pm}$issuing from $\lambda_{k}$.

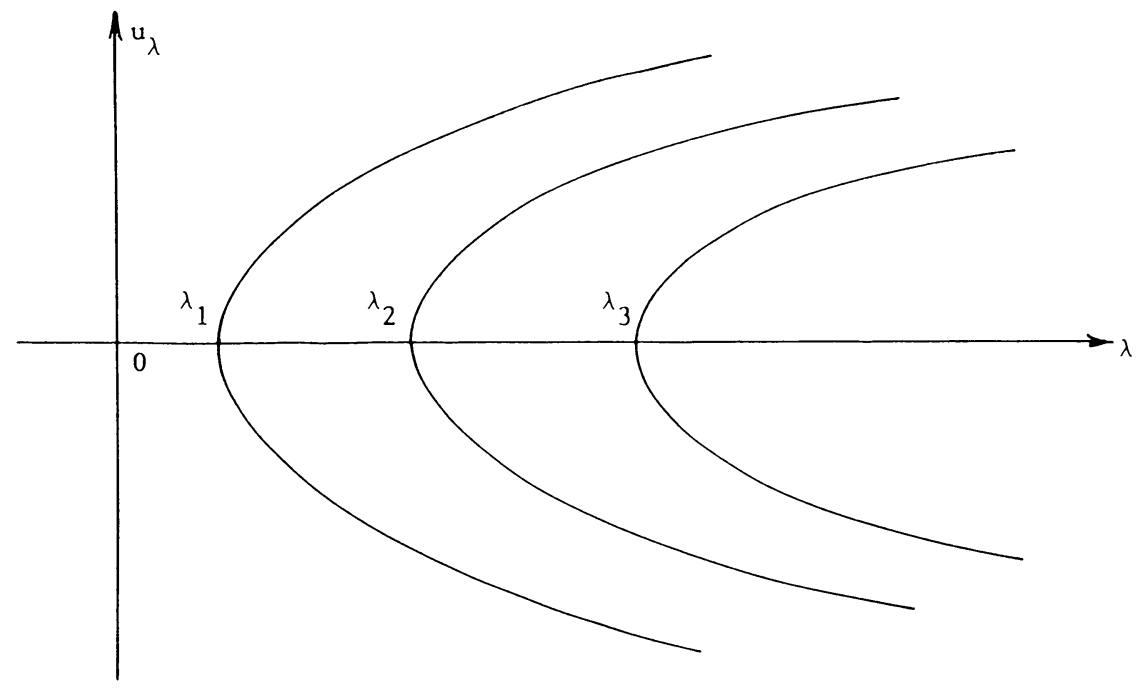

FIGURE 1

In the case $p>2$ the main difference will come from the fact that the following integral

$$
x(\lambda)=\int_{0}^{h(\lambda)} \frac{d s}{\left(\alpha^{p}(\lambda)+\frac{p}{p-1} F(s)-\frac{\lambda}{p-1} s^{p}\right)^{1 / p}}
$$

is finite as $h(\lambda)$ is a double zero of $\Psi(\alpha(\lambda), r)$.

THEOREM 2.2. Assume $p>2$ and (2.2)-(2.7). Then

(i) if $\lambda \leq \lambda_{1} E_{\lambda}=\{0\}$,

(ii) if $\lambda_{k}<\lambda \leq \lambda_{k+1}$ for some $k \in \mathbf{N}^{*}$

$$
E_{\lambda}=\{0\} \cup\left\{ \pm u_{1}\right\} \bigcup_{l=2}^{k}\left\{ \pm E_{\lambda}^{l}\right\}
$$


where $u_{1} \in S_{1}^{+}$and $E_{\lambda}^{l} \subset S_{l}^{+}, l=2, \ldots, k$, and

$E_{\lambda}^{l}$ is reduced to a single element if $2 l x(\lambda) \geq 1$,

$E_{\lambda}^{l}$ is diffeomorphic to $[0,1]^{l-1}$ if $0<2 p x(\lambda)<1 .^{1}$

PROOF. The idea is essentially the same as in Theorem 2.1 except that in Step 2, Case 2 (that is, if $\alpha^{p}=\alpha^{p}(\lambda)$ ) gives rise to solutions of (2.1) with maximum value $h(\lambda)$, and in that case Serrin and Veron's existence and uniqueness result does not apply; moreover the value $u=h(\lambda)$ is a bifurcation value for (2.1).

Step 1. Assume $2 x(\lambda) \geq 1$. Then the construction of Theorem 2.1 works: the function $\alpha \mapsto 1 / 2 \theta(\alpha)$ is a decreasing diffeomorphism from $(0, \alpha(\lambda)]$ onto $\left[1 / 2 x(\lambda),\left(\lambda / \lambda_{1}\right)^{1 / p}\right)$. As $\lambda_{k}<\lambda \leq \lambda_{k+1}$ there exist exactly $k$ integers $1,2, \ldots, k$ and $k$ positive real numbers $\alpha_{1}, \ldots, \alpha_{k}$ such that $1 / 2 \theta\left(\alpha_{l}\right)=l \in\left[1 / 2 x(\lambda),\left(\lambda / \lambda_{1}\right)^{1 / p}\right)$, $l=1, \ldots, k$. and we get the corresponding solutions $u_{l} \in S_{l}^{+}$by (2.26).

Step 2. Assume $4 x(\lambda) \geq 1>2 x(\lambda)$. All the elements $u_{l}=2, \ldots, k$ in $S_{l}^{+}$are constructed as in Step 1. As for the element $u_{1} \in S_{1}^{+}$it has necessarily the following form as the initial slope must be $\alpha(\lambda)$ :

$$
\begin{gathered}
\text { for } 0 \leq x \leq x(\lambda) \\
x=\int_{0}^{u_{1}(x)} \frac{d t}{(\Psi(\alpha(\lambda), t))^{1 / p}}, \\
\text { for } x(\lambda) \leq x \leq 1-x(\lambda) \\
\quad u_{1}(x)=h(\lambda), \\
\text { for } 1-x(\lambda) \leq x \leq 1 \\
x-(1-x(\lambda))=-\int_{u_{1}(x)}^{h(\lambda)} \frac{d t}{(\Psi(\alpha(\lambda), t))^{1 / p}} .
\end{gathered}
$$

Step 3. Assume $0<2 l x(\lambda)<1$ for some $l \in\{2, \ldots, k\}$. We can construct all the elements of $E_{\lambda} \cap S_{l}^{+}$in the following way as their initial slope is necessarily $\alpha(\lambda)$ :

$$
\begin{aligned}
& \text { for } 0 \leq x \leq x(\lambda) \\
& \qquad x=\int_{0}^{u_{l}(x)} \frac{d t}{(\Psi(\alpha(\lambda), t))^{1 / p}} \\
& \text { for } x(\lambda) \leq x \leq x_{1} \text { where } x_{1} \in(x(\lambda), 1) \text { and } \\
& \quad x_{1}-x(\lambda) \leq 1-2 l x(\lambda) \\
& \quad \text { then } u_{l}(x)=h(\lambda) \\
& \text { for } x_{1} \leq x \leq 2 x(\lambda)+x_{1}
\end{aligned}
$$

$$
x-x_{1}=-\int_{u_{l}(x)}^{h(\lambda)} \frac{d t}{(\Psi(\alpha(\lambda), t))^{1 / p}},
$$

for $x_{1}+2 x(\lambda) \leq x \leq x_{2}$ where $x_{2} \in\left(x_{1}+2 x(\lambda), 1\right)$ and

$$
\begin{aligned}
& x_{2}-\left(x_{1}+2 x(\lambda)\right)+x_{1}-x(\lambda) \leq 1-2 l x(\lambda) \\
& \text { then } u_{l}(x)=-h(\lambda) .
\end{aligned}
$$

${ }^{1}$ And more naturally to the set $K_{l}=\left\{x=\left(x^{1}, \ldots, x^{l}\right), x^{j} \geq 0, \sum_{j=1}^{l} x^{j}=1-2 l x(\lambda)\right\}$. 
Continuing this procedure any solution $u_{l} \in S_{l}^{+}$is defined by the intervals $I_{j}=$ $\left[x_{j-1}+2 x(\lambda), x_{j}\right], j=1, \ldots, l$, and $x_{0}=-x(\lambda)$ where it takes the constant value $(-1)^{j+1} h(\lambda)$ and the intervals $\left[x_{j-1}, x_{j-1}+2 x(\lambda)\right]$ where it is defined by

$$
x-x_{j-1}=-\int_{u_{l}(x)}^{h(\lambda)} \frac{d t}{(\Psi(\alpha(\lambda), t))^{1 / p}}
$$

if $j$ is even or

$$
x-x_{j-1}=\int_{h(\lambda)}^{u_{l}(x)} \frac{d t}{(\Psi(\alpha(\lambda), t))^{1 / p}}
$$

if $j$ is odd.

From the above construction the total length of the $I_{j}$ is $1-2 l x(\lambda)$ and the set $E_{\lambda}^{l}$ of the $u_{l}$ is diffeomorphic to the $(l-1)$-dimensional cube.

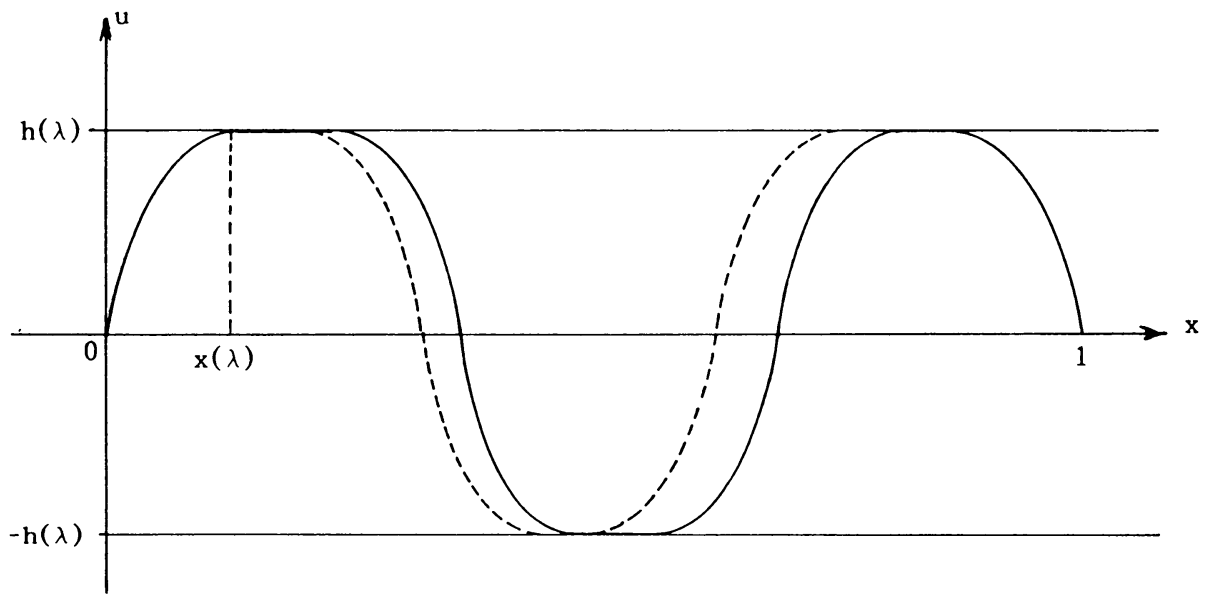

FIGURE 2. Example of construction of $E_{\lambda}^{3}$

REMARK 2.3. It is important to notice that this type of secondary bifurcation along the branch of solutions issuing from $\lambda_{k}, k \geq 2$, always appears if we have

$$
\lim _{\lambda \rightarrow+\infty} x(\lambda)=0 \text {. }
$$

This is in particular the case if $f(r) \underset{r \rightarrow+\infty}{\sim}|r|^{q-1} r$ which implies

$$
x(\lambda) \underset{\lambda \rightarrow+\infty}{\sim} \lambda^{-1 / p} \int_{0}^{1}\left(\frac{q+1-p}{(p-1)(q+1)}+\frac{p}{(p-1)(q+1)} \sigma^{q+1}-\frac{\sigma^{p}}{p-1}\right)^{-1 / p} d \sigma .
$$

However this is not always the case under conditions (2.2)-(2.7), for example, with $f(r)=\left(|r|^{p-2} \log |r|\right) r$ for $|r| \geq 2$, where we get

$$
\lim _{\lambda \rightarrow+\infty} x(\lambda)=\int_{0}^{1}\left(\frac{1}{p(p-1)}\left(1-\sigma^{p}\right)+\frac{1}{p-1} \sigma^{p} \log \sigma\right)^{-1 / p} d \sigma
$$

We finally have the following exclusion principle. 
THEOREM 2.3. Assume $p>1,(2.2)-(2.7), g$ is a continuous even function increasing on $\mathbf{R}^{+}$and $u_{1}$ and $u_{2}$ are two solutions of (2.1); then

(i) if $u_{1}$ and $u_{2}$ have the same number of zeros

$$
\int_{0}^{1} g\left(u_{1}(x)\right) d x=\int_{0}^{1} g\left(u_{2}(x)\right) d x
$$

(ii) if $u_{1}$ and $u_{2}$ do not have the same number of zeros

$$
\int_{0}^{1} g\left(u_{1}(x)\right) d x \neq \int_{0}^{1} g\left(u_{2}(x)\right) d x .
$$

PROOF. It is clear that for any function $\int_{0}^{1} g(u(x)) d x$ is equal to $\int_{0}^{1} g(-u(x)) d x$. When $p>2$ we have only to consider two solutions of $E_{\lambda}$ with the same number of zeros and belonging to some $E_{\lambda}^{l}, l \geq 2$, in the case $2 l x(\lambda)<1$. In that case $u_{1}$ and $u_{2}$ take the value $\pm h(\lambda)$ on $l$ intervals $I_{j}^{1}$ and $I_{j}^{2}, j=1, \ldots, l$, which are disconnected and have the same total length which gives

$$
\int_{\bigcup_{j} I_{j}^{1}} g\left(u_{1}(x)\right) d x=\int_{\bigcup_{j} I_{j}^{2}} g\left(u_{2}(x)\right) d x=(1-2 l x(\lambda)) g(h(\lambda)) .
$$

On $(0,1) \backslash\left\{\bigcup_{j} I_{j}^{1}\right\}$ or $(0,1) \backslash\left\{\bigcup_{j} I_{j}^{2}\right\} u_{1}$ and $u_{2}$ are defined by the same types of formula ((2.32) or (2.30)) and the integral of $g\left(u_{i}\right)$ over these sets is

$$
2 l \int_{0}^{x(\lambda)} g\left(u_{1}(x)\right) d x
$$

Hence, for $i=1,2$, we get

$$
\int_{0}^{1} g\left(u_{i}(x)\right) d x=(1-2 l x(\lambda)) g(h(\lambda))+2 l \int_{0}^{x(\lambda)} g\left(u_{i}(x)\right) d x
$$

which proves (i).

For proving (ii) we shall assume either $1<p \leq 2$ or $p>2$ but $u_{1}$ and $u_{2}$ are not constant on any subinterval of $(0,1)$ (the other case is essentially the same). If $u_{1}$ and $u_{2}$ do not have the same number of zeros in $(0,1)$ we can assume $u_{1 x}(0)=\alpha$, $u_{2 x}(0)=\beta, 0<\alpha<\beta ; u_{1}$ is $4 \theta(\alpha)$-periodic, $u_{2}$ is $4 \theta(\beta)$-periodic and $0<\theta(\alpha)<$ $\theta(\beta)$. Moreover

$$
\frac{1}{2 \theta(\alpha)}=k_{1}, \quad \frac{1}{2 \theta(\beta)}=k_{2}, \quad k_{1}, k_{2} \in \mathbf{N}^{*}, k_{1}>k_{2} .
$$

Step 1 . For $0<x<\theta(\alpha)$ we have $0<u_{1}(x)<u_{2}(x)$. On a right neighbourhood of 0 we have $u_{1}<u_{2}$, and $u_{1}$ and $u_{2}$ are increasing on $[0, \theta(\alpha)]$. If we assume the existence of some $x_{0} \in[0, \theta(\alpha)]$ such that $u_{1}\left(x_{0}\right)=u_{2}\left(x_{0}\right)$, we can always suppose that $u_{1}<u_{2}$ in $\left(0, x_{0}\right)$ and then $u_{1 x}\left(x_{0}\right) \geq u_{2 x}\left(x_{0}\right)$. The energy relation implies

$$
\begin{aligned}
\alpha^{p}+ & \frac{p}{p-1} F\left(u_{1}\left(x_{0}\right)\right)-\frac{\lambda}{p-1} u_{1}^{p}\left(x_{0}\right) \\
& \geq \beta^{p}+\frac{p}{p-1} F\left(u_{2}\left(x_{0}\right)\right)-\frac{\lambda}{p-1} u_{2}^{p}\left(x_{0}\right)
\end{aligned}
$$

and $\alpha \geq \beta$ which is impossible. 
Step 2. End of the proof. From Step 1: $0<u_{1}(x)<u_{2}\left(x^{\prime}\right)$ for $0<x<\theta(\alpha)$ and $0<x \leq x^{\prime}<\theta(\beta)$. Set $\varphi$ the lowest common multiple to $k_{1}$ and $k_{2}$. There exist $n_{1}$ and $n_{2} \in \mathbf{N}^{*}$ such that $n_{1} k_{1}=n_{2} k_{2}=\varphi$ and

$$
n_{1} / \theta(\alpha)=n_{2} / \theta(\beta), \quad 0<n_{1}<n_{2} .
$$

Then

$$
\begin{aligned}
& \int_{0}^{1} g\left(u_{1}(x)\right) d x=\frac{1}{\theta(\alpha)} \int_{0}^{\theta(\alpha)} g\left(u_{1}(x)\right) d x, \\
& \int_{0}^{1} g\left(u_{2}(x)\right) d x=\frac{1}{\theta(\beta)} \int_{0}^{\theta(\beta)} g\left(u_{2}(x)\right) d x .
\end{aligned}
$$

Setting $T=n_{2} \theta(\alpha)=n_{1} \theta(\beta)$, we have

$$
\begin{aligned}
\frac{1}{\theta(\alpha)} \int_{0}^{\theta(\alpha)} g\left(u_{1}(x)\right) d x & =\frac{1}{n_{2} \theta(\alpha)} \int_{0}^{n_{2} \theta(\alpha)} g\left(u_{1}\left(\frac{\sigma}{n_{2}}\right)\right) d \sigma \\
& =\frac{1}{T} \int_{0}^{T} g\left(u_{1}\left(\frac{\sigma}{n_{2}}\right)\right) d \sigma
\end{aligned}
$$

and

$$
\frac{1}{\theta(\beta)} \int_{0}^{\theta(\beta)} g\left(u_{2}(x)\right) d x=\frac{1}{T} \int_{0}^{T} g\left(u_{2}\left(\frac{\sigma}{n_{1}}\right)\right) d \sigma
$$

which implies

$$
\int_{0}^{1} g\left(u_{1}(x)\right) d x<\int_{0}^{1} g\left(u_{2}(x)\right) d x .
$$

REMARK 2.4. As a consequence there exist $k+1$ different critical values for the energy functional

$$
J(\omega)=\frac{1}{p} \int_{0}^{1}\left|\omega_{x}\right|^{p} d x+\int_{0}^{1} F(\omega) d x-\frac{\lambda}{p} \int_{0}^{p}|\omega|^{p} d x
$$

defined in $W_{0}^{1, p}(0,1)$, for $\lambda_{k}<\lambda \leq \lambda_{k+1}$; those critical values only depend on the set $S_{l}, l=1, \ldots, k$, the critical points of $(2.52)$ belong to. This is an immediate consequence of Theorem 2.3 and the fact that

$$
J(u)=\int_{0}^{1}\left(F(u)-\frac{1}{p} u f(u)\right) d x
$$

for $u \in E_{\lambda}$.

\section{REFERENCES}

1. H. Berestycki, Le nombre de solutions de certains problèmes semi-linéaires elliptiques, J. Funct. Anal. 40 (1981), 1-19.

2. M. S. Berger, Nonlinearity and functional analysis, Academic Press, 1977.

3. X. Y. Chen, H. Matano and L. Veron, Anisotropic singularities of solutions of nonlinear elliptic equations in $\mathbf{R}^{2}$, J. Funct. Anal. (to appear).

4. M. Guedda and L. Veron, Quasilinear elliptic equations involving critical Sobolev exponents, Nonlinear Anal. (to appear). 
5. M. Otani, On certain second order differential equations associated with Sobolev-Poincaré-type inequalities, Nonlinear Anal. 8 (1984), 1255-1270.

6. F. De Thelin, Quelques resultats d'existence et de non-existence pour une E. D. P. elliptique non linéraire, C. R. Acad. Sci. Paris 299 (1984), 911-914.

7. __ Sur l'espace propre associé à la première valeur propre du pseudo-laplacien, C. R. Acad. Sci. Paris 303 (1986), 355-358.

Département de Mathématiques, Faculté des Sciences, Parc de Grandmont, F-37200 TOURS, FRANCE 